PROCEEDINGS OF THE

AMERICAN MATHEMATICAL SOCIETY

Volume 134, Number 3, Pages 723-730

S 0002-9939(05)08227-4

Article electronically published on August 29, 2005

\title{
GLOBAL APPROXIMATION OF CR FUNCTIONS ON BLOOM-GRAHAM MODEL GRAPHS IN $\mathbb{C}^{n}$
}

\author{
ALBERT BOGGESS AND DANIEL JUPITER
}

(Communicated by Mei-Chi Shaw)

\begin{abstract}
We define a class of generic CR submanifolds of $\mathbb{C}^{n}$ of real codimension $d, 1 \leq d \leq n$, called the Bloom-Graham model graphs, whose graphing functions are partially decoupled in their dependence on the variables in the real directions. We prove a global version of the Baouendi-Treves CR approximation theorem for Bloom-Graham model graphs with a polynomial growth assumption on their graphing functions.
\end{abstract}

\section{INTRODUCTION}

Baouendi and Treves 1 proved that CR functions on a generic CR submanifold of $\mathbb{C}^{n}$ can be locally approximated by entire functions. This theorem cannot in general be extended to a global result. For example, the function $f(z, w)=1 / z$ on the CR manifold $\left\{(z, w) \in \mathbb{C}^{2} ;|z|=1\right\}$ cannot be uniformly approximated on compacts by entire functions.

There are, however, results indicating that global approximation is possible in certain situations where there are no topological obstructions. For example, Boggess and Dwilewicz [4] showed that continuous CR functions on hypersurface graphs can be uniformly approximated on compacts by entire functions. The problem is more complicated in higher codimension. Dwilewicz and Gauthier [5] have proved global approximation results in this case. Their results require certain convexity restrictions. Nunemacher [6] similarly proved a global approximation result, for the case of totally real submanifolds. For rigid graphs, where the graphing function is independent of the totally real coordinates, certain global CR approximation results are known. (See e.g. [3, where approximation in $L^{p}$-norm is established.) In this work, we establish global CR approximation results on a class of graphs which contain the rigid ones, under an additional assumption that the graphing functions satisfy a polynomial growth condition. The class under consideration, called the Bloom-Graham model graphs, is defined as follows.

Definition 1.1. Let $M$ be a generic CR submanifold of $\mathbb{C}^{n}$ of codimension $d$, $1 \leq d \leq n$.

Received by the editors October 4, 2004.

2000 Mathematics Subject Classification. Primary 32V10, 32V99, 30E10.

Key words and phrases. CR approximation, Bloom-Graham model graphs. 
Suppose the coordinates of $\mathbb{C}^{n}$ are given by $(z, w) \in \mathbb{C}^{d} \times \mathbb{C}^{n-d}$,

$$
\begin{aligned}
z_{j} & =x_{j}+i y_{j}, \quad j=1, \ldots, d, \\
w_{j} & =u_{j}+i v_{j}, \quad j=1, \ldots, n-d .
\end{aligned}
$$

$M$ is a Bloom-Graham model graph if $M$ is given globally as a graph of the form

$$
M=\left\{(z, w) \in \mathbb{C}^{d} \times \mathbb{C}^{n-d} ; y_{j}=h_{j}\left(x_{1}, \ldots, x_{j-1}, w\right), j=1, \ldots, d\right\},
$$

where

$$
h=\left(h_{1}, \ldots, h_{d}\right): \mathbb{R}^{d} \times \mathbb{C}^{n-d} \rightarrow \mathbb{R}^{d}
$$

is a $C^{1}$ map.

Note that the graphing functions are partially decoupled in their dependence on the variables in the real directions, in the sense that $h_{j}$ depends only on $x_{1}, \ldots, x_{j-1}$. Additionally, these graphing functions look similar to the lower order terms in the Bloom-Graham normal form for a CR manifold [2]. We now state our main theorem.

Theorem 1.2. Let $M$ be a Bloom-Graham model graph, as above. Assume that

$$
|D h(x, w)| \leq C\left(1+|x|^{N}+|w|^{N}\right),
$$

for all $x \in \mathbb{R}^{d}, w \in \mathbb{C}^{n-d}$, where $D$ denotes the real derivative, and where $N$ and $C$ are uniform constants. Let $K$ be a compact subset of $M$. Then there exists a compact subset, $K^{\prime}$, of $M$ with $K \subset K^{\prime}$, such that if $f$ is a continuous $C R$ function on a neighbourhood of $K^{\prime}$, then there is a sequence of entire functions which converge to $f$ uniformly on $K$.

\section{OutLine OF The PROOF}

As with the proof of Baouendi and Treves' local approximation result, our proof proceeds by using a convolution kernel which is entire, and which is integrated along a totally real $n$-dimensional slice of $M$. In the proof below, we assume $d<n$. The case when $d=n$ is similar and easier.

For a point $p=(z, w)=(x+i h(x, w), u+i v) \in M$ define the slice

$$
M_{p}=M_{v}=\{(\zeta, \eta) \in M ; \operatorname{Im} \eta=v\} .
$$

The kernel we use is somewhat different than the Gaussian used by Baouendi and Treves. For $\zeta \in \mathbb{C}^{d}, \eta \in \mathbb{C}^{n-d}$ define

$$
\widetilde{E}(\zeta, \eta)=\zeta_{d}^{2}+\sum_{j=1}^{d-1} \Lambda_{j}\left(\zeta_{j}^{2}+\zeta_{j}^{P_{j}}\right)+\Gamma\left(\sum_{j=1}^{n-d} \eta_{j}^{2}+\eta_{j}^{Q}\right),
$$

where the constants $\Lambda_{j}, P_{j}, \Gamma$ and $Q$ are positive integers which will be chosen later, and which will depend only on the given compact $K$.

For $\epsilon>0$, let

$$
E_{\epsilon}(\zeta, \eta)=\frac{\widetilde{E}(\epsilon \zeta, \epsilon \eta)}{\epsilon^{2}}=\zeta_{d}^{2}+\sum_{j=1}^{d-1} \Lambda_{j}\left(\zeta_{j}^{2}+\epsilon^{P_{j}-2} \zeta_{j}^{P_{j}}\right)+\Gamma\left(\sum_{j=1}^{n-d} \eta_{j}^{2}+\epsilon^{Q-2} \eta_{j}^{Q}\right) .
$$

For $R>0$ large enough, let $\chi_{R}: \mathbb{R}^{d} \times \mathbb{R}^{n-d} \rightarrow \mathbb{R}$ be a smooth cutoff function which is 1 on $\{|x|+|u| \leq R\} \supset K$ and vanishes outside of $\{|x|+|u| \leq R+1\}$. 
Suppose $f$ is a continuous function defined on $M$; for $p=(z, w)=(x+i y$, $u+i v) \in \mathbb{C}^{d} \times \mathbb{C}^{n-d}$ define

$$
\begin{array}{r}
G_{\epsilon}(f)(z, w)=\frac{1}{C_{1} \epsilon^{n}} \int_{(\zeta, \eta) \in M_{v}} \chi_{R}(\operatorname{Re} \zeta, \operatorname{Re} \eta) f(\zeta, \eta) \\
\exp \left(-E_{\epsilon}\left(\left(\frac{\zeta-z}{\epsilon}\right),\left(\frac{\eta-w}{\epsilon}\right)\right)\right) d \zeta \wedge d \eta,
\end{array}
$$

where $C_{1}$ is a normalizing constant, to be chosen later.

We will show (Section 4) that $G_{\epsilon}(f) \rightarrow f$ on $K$, as $\epsilon \rightarrow 0$. Note that the integrand defining $G_{\epsilon}(f)(z, w)$ is holomorphic in $z$ and $w$. However, $G_{\epsilon}(f)(z, w)$ is not necessarily holomorphic in $(z, w)$ since the domain of integration $M_{v}$ depends on $v=\operatorname{Im} w$. Later (Section [5), we will show that if $f$ is CR on a suitable subset, $K^{\prime}$, of $M$, then the domain of integration, $M_{v}$, can be fixed, independent of $w$. The resulting sequence of functions, denoted $F_{\epsilon}(f)(z, w)$, will be entire and will converge to $f$ uniformly on $K$ as $\epsilon \rightarrow 0$.

\section{Kernel estimates}

To prove that $G_{\epsilon}(f) \rightarrow f$, we begin by pulling back the integral defining $G_{\epsilon}(f)$ from $M_{v}$ to $\mathbb{R}^{d} \times \mathbb{R}^{n-d}$, via the map $H^{v}: \mathbb{R}^{d} \times \mathbb{R}^{n-d} \rightarrow M_{v}$ :

$$
\begin{aligned}
H^{v}(s, t) & =\left(\zeta^{v}(s, t), \eta^{v}(s, t)\right) \\
& =(s+i h(s, t+i v), t+i v),
\end{aligned}
$$

for $(s, t) \in \mathbb{R}^{d} \times \mathbb{R}^{n-d}$. We obtain

$$
\begin{aligned}
G_{\epsilon}(f)(z, w)= & \frac{1}{C_{1} \epsilon^{n}} \int_{(s, t) \in \mathbb{R}^{d} \times \mathbb{R}^{n-d}} \chi_{R}(s, t) f\left(H^{v}(s, t)\right) \\
& \exp \left(-E_{\epsilon}\left(\left(\frac{\zeta^{v}(s, t)-z}{\epsilon}\right),\left(\frac{\eta^{v}(s, t)-w}{\epsilon}\right)\right)\right) d s d t .
\end{aligned}
$$

Note that since $M$ is a Bloom-Graham graph, $\left(H^{v}\right)^{*}(d \zeta \wedge d \eta)=d s \wedge d t$.

We now need a key estimate on the exponent of our kernel, which we will state in terms of $\widetilde{E}(\zeta, \eta)$, since $E_{\epsilon}(\zeta / \epsilon, \eta / \epsilon)=\widetilde{E}(\zeta, \eta) / \epsilon^{2}$.

Lemma 3.1. Let $M$ and $K$ be as in Theorem 1.2. There exist positive even integers $\Lambda_{1}, \ldots, \Lambda_{d-1}, P_{1}, \ldots, P_{d-1}, \Gamma, Q$, and a positive real constant $\widetilde{C}$, all of which depend only on $K$, such that

$$
\begin{aligned}
& -\operatorname{Re} \widetilde{E}\left(\zeta^{v^{\prime}}(s, t)-z, \eta^{v^{\prime}}(s, t)-w\right) \\
& \leq-\frac{\left(s_{d}-x_{d}\right)^{2}}{2}-\sum_{j=1}^{d-1}\left(\left(s_{j}-x_{j}\right)^{2}+\left(s_{j}-x_{j}\right)^{P_{j}}\right) \\
& \quad-\sum_{j=1}^{n-d}\left(\left(t_{j}-u_{j}\right)^{2}+\left(t_{j}-u_{j}\right)^{Q}\right)+\widetilde{C}\left(\sum_{j=1}^{n-d}\left(\left(v_{j}^{\prime}-v_{j}\right)^{2}+\left(v_{j}^{\prime}-v_{j}\right)^{Q}\right)\right),
\end{aligned}
$$

for all $(z, w)=(x+i h(x, w), u+i v) \in K$ and all $\left(s, t+i v^{\prime}\right)$ in $\mathbb{R}^{d} \times \mathbb{C}^{n-d}$. 
Remark 3.2. To show that $G_{\epsilon}(f) \rightarrow f$ in Section 4 , we will only need the above estimate with $v^{\prime}=v$. In order to fix the domain of integration in Section 5, we will need the above estimate with $v^{\prime} \neq v$.

Proof. For $\left(\zeta^{v^{\prime}}, \eta^{v^{\prime}}\right)=\left(\zeta^{v^{\prime}}(s, t), \eta^{v^{\prime}}(s, t)\right)$ and $(z, w) \in M$, we have

$$
\zeta_{j}^{v^{\prime}}-z_{j}=\left(s_{j}-x_{j}\right)+i\left[h_{j}\left(s_{1}, \ldots, s_{j-1}, t, v^{\prime}\right)-h_{j}\left(x_{1}, \ldots, x_{j-1}, u, v\right)\right] .
$$

We estimate the imaginary part by using the mean value theorem and the assumed polynomial growth estimate on $|D h|$ to obtain

$$
\begin{aligned}
\left|h_{j}\left(s_{1}, \ldots, s_{j-1}, t, v^{\prime}\right)-h_{j}\left(x_{1}, \ldots, x_{j-1}, u, v\right)\right| \\
\leq C\left(C_{K}+\sum_{k=1}^{j-1}\left|s_{k}-x_{k}\right|^{N}+|t-u|^{N}+\left|v^{\prime}-v\right|^{N}\right) \\
\cdot\left(|t-u|+\left|v^{\prime}-v\right|+\sum_{k=1}^{j-1}\left|s_{k}-x_{k}\right|\right),
\end{aligned}
$$

for all $\left(s, t+i v^{\prime}\right) \in \mathbb{R}^{d} \times \mathbb{C}^{n-d}$ and all $(z, w) \in K$.

Note that we have used the fact that if $K \subset \mathbb{R}^{d}$ is compact, then there is a constant $C_{K}$ such that

$$
1+\left|s^{*}\right|^{N} \leq C_{K}\left(1+|s-x|^{N}\right)
$$

for all $s \in \mathbb{R}^{d}, x \in K$, and $s^{*} \in \mathbb{R}^{d}$ lying between $x$ and $s$.

We will now make repeated use of a standard arithmetic inequality. Fix any $p, q>1$ with $\frac{1}{p}+\frac{1}{q}=1$ and fix any small $\delta>0$. Then there is a large constant $L_{\delta}$ with

$$
a b \leq \delta a^{p}+L_{\delta} b^{q}
$$

for any $a, b \geq 0$. A standard special case is $p=q=2$ and $\delta=L_{\delta}=1 / 2$.

Using (3.2) and (3.3), and the above arithmetic inequality, we see that if $P$ is an even integer, then there exists a positive integer $M=M_{P}$, depending only on $P$ and $N$, such that

$$
\begin{aligned}
& -\operatorname{Re}\left(\zeta_{j}^{v^{\prime}}(s, t)-z_{j}\right)^{P} \leq-\frac{\left(s_{j}-x_{j}\right)^{P}}{2} \\
& \quad+C_{K}\left[\sum_{k=1}^{j-1}\left(\left|s_{k}-x_{k}\right|^{2}+\left|s_{k}-x_{k}\right|^{M}\right)+|t-u|^{2}+|t-u|^{M}\right. \\
& \left.\quad+\left|v^{\prime}-v\right|^{2}+\left|v^{\prime}-v\right|^{M}\right] .
\end{aligned}
$$

(Inequality (3.4) is used to handle cross terms in the expansion of $\left(\zeta_{j}^{v^{\prime}}-z_{j}\right)^{P}$.) Here and below, $C_{K}$ is a constant which depends only on $K$ and may change from line to line.

Let $j=d$ and $P=2$ in (3.5). The term $-\left(s_{d}-x_{d}\right)^{2} / 2$ on the right side of (3.5) is the first term on the right side of our desired inequality (3.1). Next, let $j=d-1$ with $P=2$, and then let $j=d-1$ with $P=P_{d-1}$, an even integer which is greater than $M=M_{2}$ in (3.5). Since the sum on the right-hand side of (3.5) does not involve $\left(s_{l}-x_{l}\right)$ for $l \geq j$, we can choose a constant $\Lambda_{d-1}>2\left(C_{K}+1\right)$ and then 
combine these three inequalities (i.e. (3.5) with $j=d, P=2 ; j=d-1, P=2$; and $j=d-1$ and $P=P_{d-1}>M_{2}$ ) to obtain

$$
\begin{aligned}
& -\operatorname{Re}\left[\left(\zeta_{d}^{v^{\prime}}(s, t)-z_{d}\right)^{2}+\Lambda_{d-1}\left[\left(\zeta_{d-1}^{v^{\prime}}(s, t)-z_{d-1}\right)^{2}\right.\right. \\
& \left.\left.\quad+\left(\zeta_{d-1}^{v^{\prime}}(s, t)-z_{d-1}\right)^{P_{d-1}}\right]\right] \\
& \leq-\frac{\left(s_{d}-x_{d}\right)^{2}}{2}-\left(s_{d-1}-x_{d-1}\right)^{2}-\left(s_{d-1}-x_{d-1}\right)^{P_{d-1}} \\
& +C_{K}\left[\sum_{k=1}^{d-2}\left(\left|s_{k}-x_{k}\right|^{2}+\left|s_{k}-x_{k}\right|^{\widehat{M}}\right)+|t-u|^{2}+|t-u|^{\widehat{M}}+\left|v^{\prime}-v\right|^{2}\right. \\
& \left.+\left|v^{\prime}-v\right|^{\widehat{M}}\right]
\end{aligned}
$$

where $\widehat{M}$ depends only on $P_{d-1}$ and $M=M_{2}$, which in turn depend only on $N$. Note that the first three terms on the right of (3.6) agree with the first three terms on the right of (3.1). Continuing in this manner, we can inductively choose even integers $\Lambda_{d-1}, P_{d-1}, \ldots, \Lambda_{1}, P_{1}$, and $M^{\prime} \geq 2$, in that order and depending only on $K$, so that

$$
\begin{aligned}
-\operatorname{Re} & {\left[\left(\zeta_{d}^{v^{\prime}}(s, t)-z_{d}\right)^{2}+\sum_{j=1}^{d-1} \Lambda_{j}\left[\left(\zeta_{j}^{v^{\prime}}(s, t)-z_{j}\right)^{2}+\left(\zeta_{j}^{v^{\prime}}(s, t)-z_{j}\right)^{P_{j}}\right]\right] } \\
& \leq \frac{-\left(s_{d}-x_{d}\right)^{2}}{2}-\left(\sum_{j=1}^{d-1}\left(s_{j}-x_{j}\right)^{2}+\left(s_{j}-x_{j}\right)^{P_{j}}\right) \\
& +C_{K}\left[|t-u|^{2}+|t-u|^{M^{\prime}}+\left|v^{\prime}-v\right|^{2}+\left|v^{\prime}-v\right|^{M^{\prime}}\right]
\end{aligned}
$$

Now write $w=u+i v, \eta^{v^{\prime}}=t+i v^{\prime}$. Using the arithmetic inequality (3.4), and with $Q$ an even integer, we obtain

$$
-\operatorname{Re}\left(\eta^{v^{\prime}}(s, t)-w\right)^{Q} \leq \frac{-(t-u)^{Q}}{2}+L\left(v^{\prime}-v\right)^{Q},
$$

where $L$ is a fixed constant. Using (3.7), and choosing $\Gamma>2\left(C_{K}+1\right)$ and $Q \geq M^{\prime}$, we obtain Lemma 3.1 .

\section{Approximation to the identity}

Lemma 4.1. If $f$ is a continuous function on $M$, then $G_{\epsilon}(f) \rightarrow f$ on $K$ as $\epsilon \rightarrow 0$.

Proof. Recall that the domain of integration of $G_{\epsilon}(f)(z, w)$ is $M_{v}=M \cap\left\{v^{\prime}=\right.$ $\operatorname{Im} \eta=v\}$, where $v=\operatorname{Im} w$.

After making the change of variables

$$
s=x-\epsilon \hat{s}, \quad t=u-\epsilon \hat{t},
$$


we obtain

$$
\begin{aligned}
& G_{\epsilon}(f)(z, w)=\frac{1}{C_{1}} \int_{(\hat{s}, \hat{t}) \in \mathbb{R}^{d} \times \mathbb{R}^{n-d}} \chi_{R}(x-\epsilon \hat{s}, u-\epsilon \hat{t}) f\left(H^{v}(x-\epsilon \hat{s}, u-\epsilon \hat{t})\right) \\
& \quad \exp \left(-E_{\epsilon}\left(\left(\frac{\zeta^{v}(x-\epsilon \hat{s}, u-\epsilon \hat{t})-z}{\epsilon}\right),\left(\frac{\eta^{v}(x-\epsilon \hat{s}, u-\epsilon \hat{t})-w}{\epsilon}\right)\right)\right) d \hat{s} d \hat{t} .
\end{aligned}
$$

Using Lemma 3.1 with $v^{\prime}=v$, and the fact that $E_{\epsilon}(\zeta / \epsilon, \eta / \epsilon)=\widetilde{E}(\zeta, \eta) / \epsilon^{2}$, we see that the real part of the exponent of our kernel is less than or equal to

$$
-\frac{\hat{s}_{d}^{2}}{2}-\sum_{j=1}^{d-1}\left(\hat{s}_{j}^{2}+\epsilon^{P_{j}-2} \hat{s}_{j}^{P_{j}}\right)-\sum_{j=1}^{n-d}\left(\hat{t}_{j}^{2}+\epsilon^{Q-2} \hat{t}_{j}^{Q}\right) \leq-\frac{\hat{s}_{d}^{2}}{2}-\sum_{j=1}^{d-1} \hat{s}_{j}^{2}-\sum_{j=1}^{n-d} \hat{t}_{j}^{2} .
$$

Since $\chi_{R} \cdot f$ is bounded, the Dominated Convergence Theorem allows us to let $\epsilon \rightarrow 0$ in the integrand of $G_{\epsilon}$. The resulting integral is

$$
\frac{1}{C_{1}} \int_{(\hat{s}, \hat{t}) \in \mathbb{R}^{d} \times \mathbb{R}^{n-d}} \chi_{R}(x, u) f\left(H^{v}(x, u)\right) \exp \left[-E_{0}\left(D H^{v}(x, u) \cdot(\hat{s}, \hat{t})^{T}\right)\right] d \hat{s} d \hat{t}
$$

where

$$
E_{0}=\zeta_{d}^{2}+\sum_{j=1}^{d-1} \Lambda_{j} \zeta_{j}^{2}+\sum_{j=1}^{n-d} \Gamma_{j} \eta_{j}^{2}
$$

and where $D$ is the usual first order derivative with respect to $x$ and $u$.

We must show that

$$
\int_{(\hat{s}, \hat{t}) \in \mathbb{R}^{d} \times \mathbb{R}^{n-d}} \exp \left[-E_{0}\left(D H^{v}(x, u) \cdot(\hat{s}, \hat{t})^{T}\right)\right] d \hat{s} d \hat{t}
$$

is a constant, independent of $x, u$, and $v$. For then by letting $C_{1}$ be this constant, the expression in (4.1) becomes $f\left(H^{v}(x, u)\right)=\chi_{R} f(z, w)=f(z, w)$ for $(z, w) \in K$, and the proof of Lemma 4.1 will be complete.

Note that since $M$ is a Bloom-Graham model graph, $D H^{v}$ is of the form

$$
\mathcal{M}(A, B)=\left(\begin{array}{cc}
I_{d \times d} & 0 \\
0 & I_{(n-d) \times(n-d)}
\end{array}\right)+\left(\begin{array}{cc}
A & B \\
0 & 0
\end{array}\right),
$$

where $I_{j}$ is the $j \times j$ identity matrix, $A$ is a $d \times d$ lower triangular matrix with zeros along the diagonal, and $B$ is a $d \times(n-d)$ matrix. In particular, $\operatorname{det} \mathcal{M}(A, B)=1$. By increasing the values of $\Lambda_{j}$ and $\Gamma_{j}$ if necessary (as in the proof of Lemma 3.1 , with only quadratic terms in the exponent), we see that

$$
\int_{(\hat{s}, \hat{t}) \in \mathbb{R}^{d} \times \mathbb{R}^{n-d}} \exp \left[-E_{0}\left(\mathcal{M}(A, B) \cdot(\hat{s}, \hat{t})^{T}\right)\right] d \hat{s} d \hat{t}
$$

is complex analytic in the entries of $A$ and $B$ on a complex neighbourhood of the region given by

$$
\left|A_{j, k}\right| \leq 2 \max _{K}\left(\left|\frac{\partial h_{j}(x, u, v)}{\partial x_{k}}\right|\right), \quad 1 \leq k<j \leq d,
$$

and

$$
\left|B_{j, k}\right| \leq 2 \max _{K}\left(\left|\frac{\partial h_{j}(x, u, v)}{\partial u_{k}}\right|\right), \quad 1 \leq j \leq d, 1 \leq k \leq n-d
$$


If $A$ and $B$ are real-valued, a change of variables of the form $(s, t)^{T}=\mathcal{M}(A, B)$. $(\hat{s}, \hat{t})^{T}$ shows that the integral in (4.3) is independent of $A$ and $B$ (again, recall that $\operatorname{det} \mathcal{M}(A, B)=1)$. By the identity theorem for holomorphic functions, the same is true when $A$ and $B$ are complex valued, belonging to the above neighbourhood. Thus (4.2) is independent of $x, u$, and $v$, as desired.

\section{Fixing The SLICE}

We have established that $G_{\epsilon}(f)(z, w) \rightarrow f(z, w)$ as $\epsilon \rightarrow 0$ for $(z, w) \in K$. While the integrand in $G_{\epsilon}$ is holomorphic in $z$ and $w$, the domain of integration depends on $v=\operatorname{Im} w$. Thus $G_{\epsilon}$ is not necessarily a holomorphic function. To remedy this defect, we fix the domain of integration at $M_{v_{0}}$, independent of $v=\operatorname{Im} w$, and define

$$
\begin{array}{r}
F_{\epsilon}(f)(z, w)=\frac{1}{C_{1} \epsilon^{n}} \int_{(\zeta, \eta) \in M_{v_{0}}} \chi_{R}(\operatorname{Re} \zeta, \operatorname{Re} \eta) f(\zeta, \eta) \\
\quad \exp \left(-E_{\epsilon}\left(\left(\frac{\zeta-z}{\epsilon}\right),\left(\frac{\eta-w}{\epsilon}\right)\right)\right) d \zeta \wedge d \eta
\end{array}
$$

with $v_{0}$ a fixed point in the projection of the compact $K$ onto the $v$-axis. As $\chi_{R} \cdot f$ is compactly supported, this integral is well defined, and thus holomorphic, for all $(z, w) \in \mathbb{C}^{n}, \epsilon>0$.

Lemma 5.1. Let $K$ be a compact subset of $M$. Let $R^{\prime}$ be any number larger than $\max _{(z, w) \in K}|v|$. Then there exists $R>0$ and $C>0$ such that if $f$ is a continuous CR function on a neighbourhood of $K^{\prime}=M \cap\left[\{|x|+|u| \leq R+1\} \times\left\{|v|<R^{\prime}\right\}\right]$, then

$$
\left|F_{\epsilon}(f)(z, w)-G_{\epsilon}(f)(z, w)\right| \leq C \epsilon,
$$

for all $(z, w) \in K$, and all $\epsilon>0$.

Since $F_{\epsilon}(f)$ is entire and $G_{\epsilon}(f) \rightarrow f$ on $K$, clearly this lemma completes the proof of Theorem 1.2 .

Proof. Consider the manifold $\widetilde{M}_{v}$ defined as

$$
\widetilde{M}_{v}=\left\{(\zeta, \eta) \in M ; \operatorname{Im} \eta=v^{\prime}=r v+(1-r) v_{0}, 0 \leq r \leq 1\right\} .
$$

$\widetilde{M}_{v}$ is an $(n+1)$ real-dimensional submanifold of $M$ with boundary components $M_{v}$ and $M_{v_{0}}$. By Stokes' theorem

$$
\begin{aligned}
G_{\epsilon}(f)(z, w) & =F_{\epsilon}(f)(z, w) \\
+ & \frac{1}{C_{1} \epsilon^{n}} \int_{(\zeta, \eta) \in \widetilde{M}_{v}} d_{(\zeta, \eta)}\left[\chi_{R}(\operatorname{Re} \zeta, \operatorname{Re} \eta) f(\zeta, \eta)\right. \\
& \left.\quad \exp \left(-E_{\epsilon}\left(\left(\frac{\zeta-z}{\epsilon}\right),\left(\frac{\eta-w}{\epsilon}\right)\right)\right) d \zeta \wedge d \eta\right] .
\end{aligned}
$$

The presence of $d \zeta \wedge d \eta$ implies that only $\bar{\partial}_{(\zeta, \eta)}$ terms appear. If $f$ is $\mathrm{CR}$ on a neighbourhood of $K^{\prime}$, then the support of the integrand is contained in $\{(\zeta, \eta)=$ $\left.(s+i h(s, t, v), t+i v) \in M ; R \leq|s|+|t| \leq R+1,|v|<R^{\prime}\right\}$. 
Since $|x|,|u|$ and $\left|v^{\prime}-v\right|$ (with $\left.v^{\prime}=r v+(1-r) v_{0}\right)$ are bounded in terms of the diameter of $K$, inequality (3.1) shows that choosing $R$ suitably large relative to the diameter of $K$ ensures that

$$
\exp \left(-E_{\epsilon}\left(\left(\frac{\zeta-z}{\epsilon}\right),\left(\frac{\eta-w}{\epsilon}\right)\right)\right) \leq e^{\frac{-R^{2}}{4 \epsilon^{2}}},
$$

for $(\zeta, \eta)$ in the support of $\bar{\partial} \chi_{R}$ and $(z, w) \in K$.

Remark 5.2. Though the assumption of $M$ being a Bloom-Graham model graph is referenced throughout this work, this assumption is only critically used in the proof of Lemma 3.1, specifically in (3.5) and (3.6).

\section{REFERENCES}

1. M. S. Baouendi and F. Treves, A property of the functions and distributions annihilated by a locally integrable system of complex vector fields, Ann. of Math. 113 (1981), 387-421. MR0607899 (82f:35057)

2. T. Bloom and I. Graham, On type conditions for generic real submanifolds of $\mathbb{C}^{n}$, Invent. Math. 40 (1977), 217-243. MR0589930 (58:28644)

3. A. Boggess, $C R$ extension for $L^{p}$ CR functions on a quadric submanifold of $\mathbb{C}^{n}$, Pac. J. Math 201 (2001), 1-18. MR:1867889(2002m:32051)

4. A. Boggess and R. Dwilewicz, CR approximation on a nonrigid hypersurface graph in $\mathbb{C}^{n}$, Pacific J. Math. 216 (2004), no. 2, 201-216. MR2094543(2005f:32059)

5. R. Dwilewicz and P. M. Gauthier, Global holomorphic approximations of CR functions on CR manifolds, Complex Variables Theory Appl. 4 (1985), 377-391. MR0858919 (88b:32041)

6. J. Nunemacher, Approximation theory on totally real submanifolds, Math. Ann. 224 (1976), 129-141. MR0422684(54:10670)

Department of Mathematics, Texas A \& M University, College Station, Texas 778433368

E-mail address: boggess@math.tamu.edu

Department of Mathematics, Texas A \& M University, College Station, Texas 778433368

E-mail address: jupiter@math.tamu.edu 